\title{
Elementos contribuintes para a aplicação da Odontologia baseada em evidências: parte I
}

\section{Contributing elements for the application of Evidence-Based Dentistry: part I}

Sinval Adalberto Rodrigues Junior*

\section{Resumo}

A Odontologia baseada em evidências (OBE) visa racionalizar a prática odontológica com base na melhor evidência científica disponível, na experiência clínica sistematizada e nas preferências e condições dos pacientes. Objetivos: este estudo apresenta elementos que fundamentam a $O B E$ e orientam a busca de evidências acerca de intervenções terapêuticas em odontologia. Revisão de literatura: a tradução da evidência gerada em pesquisas para a aplicabilidade clínica é fundamental para respaldar o atendimento clínico e é mediada por revisões sistemáticas de literatura. Essas revisões podem ser feitas em estudos primários in vitro ou em ensaios clínicos controlados randomizados (ECCRs). A busca por referências que apresentem as evidências de dado assunto é construída a partir de uma pergunta estruturada com o tipo de paciente/problema/população, a intervenção, as comparações entre alternativas e o desfecho esperado. A(s) estratégia(s) de busca deve(m) ser aplicada(s) a mais de uma base de dados de referências, incluindo necessariamente $o$ Medline-PubMed e a Biblioteca Cochrane de revisões sistemáticas e ECCRs. Quando a pergunta visa responder a uma dúvida terapêutica, os maiores níveis de evidência são apresentados por revisões sistemáticas de literatura e ECCRs. Considerações finais: a OBE é um movimento irreversível sobre como pensar o cuidado com a saúde e, portanto, deve ser abordada em todas as áreas de atuação da odontologia, tanto na graduação quanto na pós-graduação. Esta revisão de literatura apresentou elementos para a construção da pergunta clínica e das estratégias de busca por alternativas terapêuticas em odontologia.

Palavras-chave: Epidemiologia. Odontologia baseada em evidências. Prática clínica baseada em evidências.

\section{Introdução}

As Diretrizes Curriculares Nacionais dos cursos de Odontologia definem a tomada de decisão no nível individual ou coletivo como uma das habilidades e competências fundamentais a serem desenvolvidas pelos egressos desse curso. Isso envolve "a capacidade de tomar decisões visando o uso apropriado, eficácia e custo/efetividade da força de trabalho, de medicamentos, de equipamentos, de procedimentos

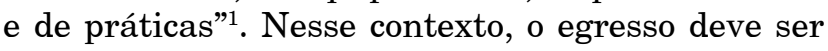
capaz de decidir a conduta mais adequada a partir da avaliação e sistematização das evidências científicas existentes ${ }^{1}$.

De modo similar, a necessidade de sistematização da melhor evidência científica para respaldar a atuação profissional em saúde foi sinalizada pelo Ministério da Saúde, que publica manuais e guias para a elaboração da síntese da evidência, pautados no modelo da prática baseada em evidência (PBE) ou da saúde baseada em evidência (SBE) ${ }^{2}$. Esse modelo, emergente na odontologia desde os anos 2000, como Odontologia baseada em evidência (OBE), existe na área médica desde a década de 1980. Sua base alia a melhor evidência científica disponível à experiência clínica e aos valores e às preferências dos pacientes.

Segundo Bernardo ${ }^{3}$ (2010), a experiência clínica sistematizada é adquirida naturalmente como um conjunto de habilidades e competências necessárias à boa prática de atendimento às necessidades de saúde. Os valores e as preferências dos pacientes são obtidos a partir do relacionamento entre o profissional da saúde e o paciente, ponderando os limites e as características culturais, estruturais e de recursos no local da assistência. Já a obtenção da melhor evidên- 
cia científica disponível demanda o desenvolvimento de habilidades específicas relacionadas à capacidade de avaliação crítica das informações publicadas. Isso exige conhecimento aprofundado na área de atuação, conhecimento estatístico, epidemiológico e dos critérios de construção de uma revisão sistemática.

A literatura científica mundial produz dados e informações a uma velocidade e em uma quantidade jamais observadas na história da humanidade ${ }^{4}$. No entanto, ao contrário do que se pensa, tamanho volume de informação não se traduz, necessariamente, em evidência de efetividade, eficácia e eficiência das terapêuticas empregadas na área da saúde.

Considerando o contexto atual, que exige o melhor nível de evidência científica subsidiando as ações e políticas de saúde e o ensino das profissões envolvidas com o cuidado em saúde, esta revisão de literatura visa fornecer elementos considerados essenciais à aplicação da OBE por professores, estudantes e cirurgiões-dentistas nas várias áreas de atuação e especialidades a fim de guiá-los na busca e avaliação da qualidade da evidência existente. Este artigo configura-se na primeira etapa de dois estudos que visam descrever o processo pelo qual a busca e a seleção de evidências podem ser estruturadas, com ênfase na busca de evidências acerca de intervenções terapêuticas em odontologia.

\section{Revisão da literatura}

A tomada de decisões clínicas por cirurgiões-dentistas é pautada por suas crenças acerca do curso da doença e da eficácia dos tratamentos, respaldada por conhecimentos da fisiologia do organismo, da etiopatogenia das doenças, do mecanismo de ação de medicamentos e de biomateriais. Esse corpo de conhecimento orienta as decisões com base na plausibilidade biológica ${ }^{5}$. Por exemplo, "se você utilizar um enxaguatório bucal com flúor associado à escovação diariamente, não desenvolverá cárie". Essa expectativa baseia-se no conhecimento do mecanismo de desenvolvimento da doença cárie, do mecanismo de ação do flúor nas estruturas dentárias e em sua substantividade.

Entretanto, a tomada de decisões com base na plausibilidade biológica tem se revelado insuficiente, trabalhosa e consumidora de tempo quando se trata da definição do melhor método de diagnóstico, prevenção, prognóstico e tratamento. O modelo frequentista, baseado na frequência de um dado evento em um grupo experimental comparado a um grupo controle, de outro modo, tem sido explorado como uma opção para lidar com os riscos e as incertezas envolvidos na decisão clínica ${ }^{5}$. Assim, no exemplo anterior, a decisão seria tomada após a observação da frequência relativa de cárie em um grupo de pacientes que utiliza enxaguatório bucal com flúor em comparação com um grupo que não o utiliza para a determinação do risco de cárie e da sua associação com o uso de enxaguatório bucal com flúor.

Esse modelo fundamenta a OBE e tem como um de seus pilares o desenho do ensaio clínico controlado randomizado (ECCR), que é considerado um dos grandes avanços da ciência moderna para a avaliação da eficácia de intervenções ${ }^{6}$. A busca vertiginosa por elementos pautados na OBE é resultado de algumas constatações: a) a necessidade sistemática e contínua de informações com consistência e validade avaliadas acerca de diagnóstico, tratamento, prognóstico e prevenção em saúde; b) a incapacidade das fontes tradicionais de prover tais informações pela desatualização, falta de acurácia ou volume excessivo; c) a impossibilidade de acessar e avaliar a qualidade da totalidade da informação disponível para aplicar em cada caso clínico, considerando suas especificidades ${ }^{7}$.

\section{Processo de translação das pesquisas para a aplicação clínica}

Entende-se a OBE como um elemento de fundamental importância para traduzir a pesquisa odontológica, em especial a pesquisa de bancada, para a prática clínica. Segundo Morris et al. ${ }^{8}$ (2011), o tempo médio decorrido para traduzir a pesquisa de bancada e implementá-la na clínica é de dezessete anos. Ainda segundo os autores, a redução desse tempo pode diminuir o custo do tratamento, que pode variar de acordo com quanto de tempo foi reduzido ${ }^{8}$.

A Figura 1 representa o fluxo de tradução da pesquisa de bancada para a aplicação clínica e destaca dois pontos nos quais o processo de tradução é sistematizado (T1 e T2). A tradução em T1 trata da compreensão de mecanismos da doença e de novos métodos de diagnóstico, tratamento e prevenção em nível laboratorial ${ }^{9}$. A adaptação do fluxograma de $\operatorname{Tracy}^{9}$ (2014) inclui em T1 a possibilidade de essa tradução ser mediada por revisões sistemáticas de literatura com ou sem metanálise, movimento comum na odontologia que desenvolve muitos estudos laboratoriais e, mais recentemente, muitas revisões sistemáticas desses estudos.

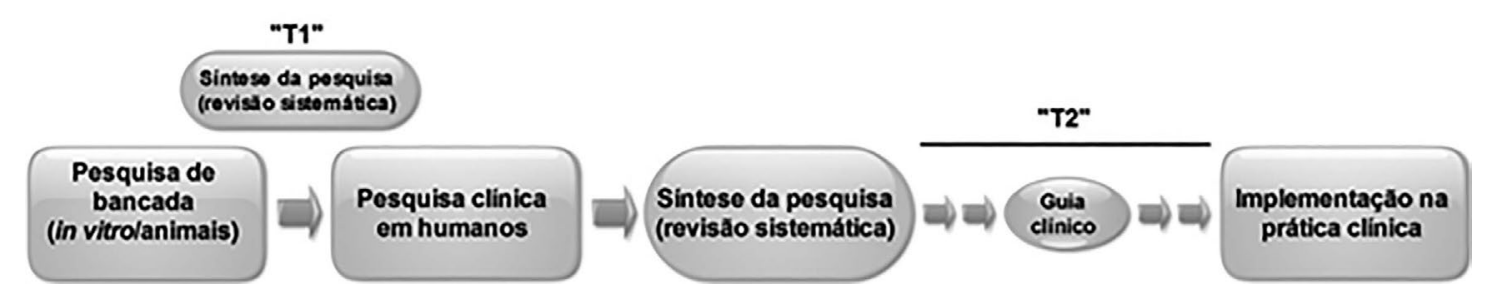

Figura 1 - Fluxograma de tradução da pesquisa de bancada para a aplicação clínica

Fonte: adaptado de Tracy $^{9}$ (2014). 
O segundo momento de tradução (T2) caracteriza-se pela sumarização de dados obtidos nas pesquisas clínicas envolvendo seres humanos também em revisões sistemáticas de literatura, embora, nesse caso, preferencialmente de ECCRs quando o desfecho for a eficácia de uma intervenção.

As revisões sistemáticas configuram-se no método que confronta toda a evidência empírica enquadrada em critérios pré-especificados de elegibilidade para responder uma questão de pesquisa específica ${ }^{10}$ e são o mecanismo de translação para o próximo passo, seja clínico ou de pesquisa ${ }^{9}$. Elas diferem das revisões descritivas em alguns aspectos: são escritas por experts no conteúdo e no método, têm uma questão de pesquisa definida claramente, utilizam métodos sistemáticos, explícitos e selecionados para minimizar vieses e acessar a qualidade metodológica dos estudos incluídos, fornecendo achados confiáveis a partir dos quais as conclusões podem ser elaboradas e as decisões tomadas ${ }^{10,11}$.

\section{Elaborando a pergunta clínica}

À luz da quantidade de artigos e de informações disponíveis na literatura atual, elaborar uma pergunta de pesquisa ou clínica de modo estruturado é de vital importância para encontrar a informação que se busca sem grande perda de tempo. A arte e a ciência da elaboração de perguntas estruturadas são desenvolvidas com a prática e deveriam ser exercitadas sempre que possível. No entanto, esse passo é muitas vezes negligenciado ou considerado de menor importância no processo de sistematização da informação disponível ${ }^{7}$. Contudo, o cenário ambulatorial médico, e mesmo o odontológico, gera inúmeras oportunidades de exercitar a transformação da necessidade de informação na elaboração de perguntas clínicas a serem respondidas com base nos princípios da $\mathrm{SBE}^{7}$.

Uma pergunta bem estruturada norteia a estratégia de busca e pode ser elaborada para verificar o efeito de uma intervenção sobre uma determinada doença ou condição, para avaliar/comparar técnicas diagnósticas, para identificar fatores etiológicos para uma dada doença ou condição e para estimar o prognóstico de uma condição sob tratamento. Para cada objetivo traçado, desenhos de estudos epidemiológicos específicos serão analisados, podendo ser ensaios clínicos controlados randomizados, estudos transversais, estudos de coorte e estudos de caso- controle ${ }^{2}$.

A estrutura de uma pergunta acerca das possibilidades diagnósticas, terapêuticas, prognósticas ou de fatores etiológicos segue o padrão sugerido pelo manual da Cochrane Collaboration ${ }^{12}$ e pelo checklist Preferred Reporting Items for Systematic Reviews and Meta-Analyses (Prisma ${ }^{10}$ e envolve, necessariamente, elementos representados pela sigla PICO. O P representa o paciente, população ou problema de interesse, o I é a principal intervenção em estudo, definida amplamente como uma exposição, um exame diagnóstico, um tratamento, o $\mathrm{C}$ representa a comparação entre intervenções, quando relevante, e o $\mathrm{O}$ (de outcome, em inglês) representa o(s) desfecho(s) clínico(s) de interesse.

Segue um exemplo de pergunta terapêutica: "Em idosos apresentando cárie ativa de raiz, qual a melhor forma de administração da fluorterapia tópica para remissão das lesões: flúor tópico gel ou verniz fluoretado?". Nessa pergunta, o P é representado pelos 'idosos apresentando cárie de raiz', o I é a 'fluorterapia tópica', o C compara o 'flúor tópico gel com o verniz fluoretado' e o $\mathrm{O}$ é compreendido como a 'remissão das lesões cariosas ativas'. Note-se que outras possibilidades terapêuticas podem ser adicionadas ao $\mathrm{C}$ e que alternativas terapêuticas experimentais podem ser comparadas com um padrão-ouro ou mesmo com a ausência de tratamento (placebo), quando pertinente.

\section{Organizando uma estratégia de busca: o que considerar?}

A estratégia de busca visa localizar todas as referências pertinentes ao assunto com potencial de gerar respostas à pergunta clínica ou de pesquisa formulada. É uma etapa bastante sensível da busca pela melhor informação, pois não é incomum encontrar estudos cujos resultados possam favorecer um grupo e outros cujo grupo favorecido é o oposto. Portanto, a não identificação de um desses estudos pode conduzir a uma conduta clínica não respaldada. Assim, também se sugere a busca em periódicos bem como a checagem das referências dos artigos identificados para assegurar que o maior número de estudos seja localizado e acessado ${ }^{13}$.

Bernardo $^{2}$ (2010) sugere iniciar a busca pela base de dados Medline-PubMed (base de acesso livre: www.pubmed.gov), já que ela traduziria melhor a questão clínica. Medline é uma base de dados da literatura internacional na área médica $\mathrm{e}$ biomédica, produzida pela National Library of Medicine (EUA). Opera com mais de 4.000 títulos de revistas publicadas nos Estados Unidos da América e em outros setenta países desde 1966 até o presente, sendo atualizada com frequência mensal ${ }^{14}$. O Medline é o correspondente computadorizado do Index Medicus e armazena informações de 3.500 revistas indexadas e 600 revistas não relacionadas no Index Medicus ${ }^{13}$. Ainda, a busca deve ser complementada pesquisando as bases Embase (base de acesso restrito, voltada à produção europeia: www. embase.com) e SciELO-LILACS (base de acesso livre, voltada à produção latino-americana: www. bvs.br). A busca também deve ser complementada na Biblioteca Cochrane (www.cochrane.bvsalud. org), que contém revisões sistemáticas e ECCRs de qualidade avaliada.

A estratégia de busca é organizada utilizando os descritores em saúde da base de dados MeSH (Me- 
dical Subject Headings), disponibilizada na página do PubMed. Nem todos os descritores que direcionam a busca constam como MeSH terms, assim, é importante incluir, além dos $\mathrm{MeSH}$ terms, os termos reconhecidos como possíveis de estreitar a busca em torno do assunto em estudo. Esses descritores são utilizados isoladamente ou em associação com a finalidade de localizar as publicações que os contêm.

Os descritores são combinados utilizando operadores booleanos (OR, AND ou NOT). O operador OR seleciona trabalhos identificados com qualquer um dos descritores, o AND seleciona, obrigatoriamente, os trabalhos que apresentam a associação dos descritores e o operador NOT exclui descritores indesejados da busca ${ }^{2}$. Recomenda-se que, dentro dos componentes do PICO, entre sinônimos, seja usado o operador OR, enquanto entre componentes, o operador usado seja o AND.

A seguir, tem-se o exemplo de duas estratégias de busca elaboradas com base na pergunta dada como exemplo:

Estratégia 1: aged OR elderly AND root caries OR caries, root OR caries, cervical OR cary, cervical OR cervical cary OR cervical caries AND caries treatment OR dental caries treatment OR root caries treatment OR non operative caries treatment OR non-operative caries treatment OR incipient caries treatment OR caries management OR root caries management AND fluoride gel OR fluoride gel application OR fluoride varnish AND caries arrest $\mathrm{OR}$ active caries arrest OR caries arrestment OR active caries arrestment

Estratégia 2: root caries management OR root caries treatment

Essas estratégias foram aplicadas em maio de 2015, a primeira no PubMed e a segunda no PubMed e na Biblioteca Cochrane.

Uma vez rastreados os estudos, o próximo passo é a seleção daqueles que podem potencialmente responder à dúvida clínica. A capacidade de um estudo gerar evidência acerca de uma alternativa terapêutica em saúde respeita a hierarquia representada na Figura 2. Assim, revisões sistemáticas de literatura fornecem o maior nível de evidência acerca da eficácia e da segurança de intervenções, seguidas por ECCRs.

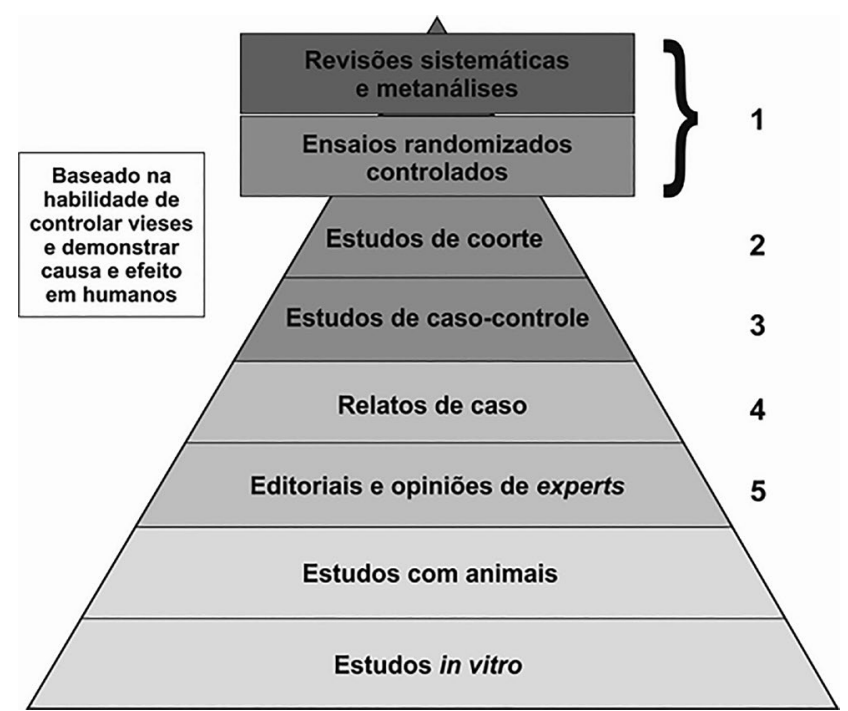

Figura 2 - Pirâmide de evidências para intervenções terapêuticas

Fonte: elaboração do autor.

Na base Medline-PubMed, o tipo de estudo pode ser selecionado: 1) com própria estratégia, incluindo o descritor do tipo de estudo que se busca ou 2) utilizando os filtros de tipos de estudo disponibilizados na página. A filtragem da busca por Systematic reviews resultou em 9 referências para a primeira estratégia e em 48 referências para a segunda. $\mathrm{Na}$ Biblioteca Cochrane, a segunda estratégia gerou 1 referência de revisão sistemática e 127 ECCRs. Observa-se que, mesmo direcionando a busca pela construção de estratégias bastante específicas, estudos fora do escopo da busca também surgem, cabendo uma seleção dos estudos pela leitura de título e resumo.

\section{Ensaios (clínicos) controlados randomizados}

O ECCR é um método de estudo prospectivo que visa atestar a eficácia de uma determinada intervenção, podendo essa intervenção significar, na área da saúde, um método, um protocolo, uma técnica, uma abordagem educativa, um material ou medicamento com finalidade terapêutica (Figura 3). Apesar de não ser o principal objetivo do estudo, a verificação dos efeitos colaterais dessa intervenção também é possível por esse modelo de estudo. A principal característica dos ECCRs é a alocação dos participantes da pesquisa em dois ou mais grupos de intervenção de modo randômico, aleatório ${ }^{6}$.

Quando bem desenhado e conduzido, o ECCR permite a verificação da eficácia de uma ou mais intervenções experimentais em comparação com um padrão-ouro, um controle de efeito reconhecido ou um placebo, pela diferença dos resultados entre os grupos (Figura 3). A geração de uma sequência randômica pode ser feita usando uma tabela de números aleatórios gerada por computador ou baseada em algum livro texto de estatística, ou mesmo por 
meio de sorteio, jogando a moeda, controlando os vieses de seleção para os grupos, uma vez que o sujeito elegível para a pesquisa tem a mesma chance de cair em qualquer um dos grupos. Desse modo, a intervenção acaba sendo o diferencial entre o sujeito de um grupo e o de outro ${ }^{6,15}$.

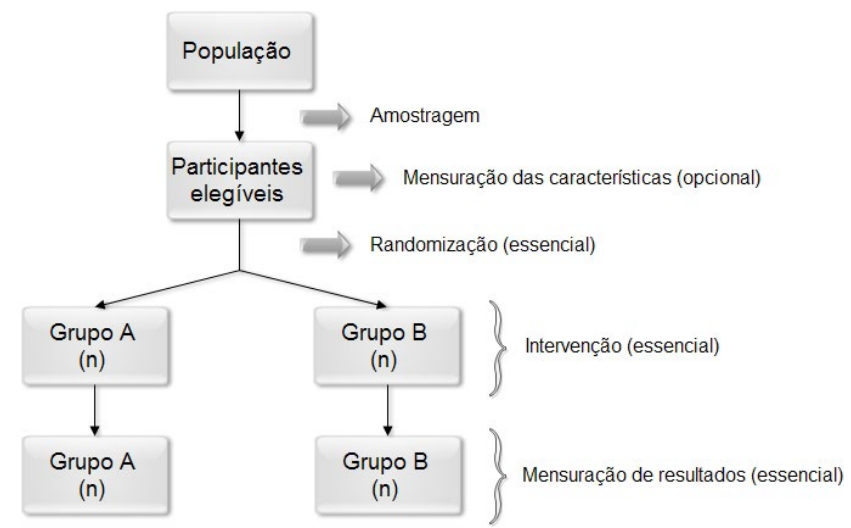

Figura 3 - Fluxograma de um ECCR

Fonte: elaboração do autor.

Outros aspectos envolvidos nos ECCRs, como o sigilo de alocação e o cegamento, embora não sejam obrigatórios para caracterizar o estudo como ensaio clínico controlado randomizado ${ }^{6}$, são fundamentais para o controle de outros vieses possíveis. Recentemente, o guia CONSORT para elaboração, execução e relato de ECCRs foi revisto e reestruturado para deixar mais explícitos os critérios necessários para atestar a qualidade metodológica desses estudos ${ }^{15}$. A possibilidade de análise crítica do risco de indução de vieses pelos ECCRs é vital para a determinação da força da evidência gerada por esses estudos, já que a incorporação dos resultados de ensaios clínicos de baixa qualidade metodológica em metanálises foi associada à alteração da interpretação dos efeitos das intervenções ${ }^{16,17}$.

Uma vez que o ECCR é o substrato de revisões sistemáticas quando seu objetivo é identificar a alternativa terapêutica mais eficaz e segura, cabe uma familiarização com esse modelo de estudo e com as possibilidades de indução de viés por parte dos cirurgiões-dentistas que visam balizar sua prática na OBE.

\section{Estudos experimentais in vitro}

Os estudos experimentais in vitro (estudos de bancada) são amplamente utilizados nas mais variadas áreas da odontologia por serem mais rápidos e de menor custo do que os ECCRs. Esses estudos constituem a base da pirâmide de evidências (Figura 2), devido a dois aspectos básicos: são produzidos em grande quantidade e geram o menor nível de evidência acerca da aplicabilidade clínica de algum procedimento.

O processo de tradução da informação obtida a partir desses estudos (T1) tem grande potencial de indicar lacunas no estado da arte do conhecimento acerca do assunto para pesquisas futuras, que tanto podem ser de bancada como podem ser pesquisas clínicas $^{9}$. Em odontologia, o esforço em efetivar o T1 resulta na produção de revisões sistemáticas com metanálise que compilam dados de estudos in vitro com a finalidade de responder a perguntas de pesquisa e indicar o próximo passo para pesquisas no assunto $^{18,19}$.

\section{Discussão}

A adoção da OBE como modelo de prática odontológica gera a tranquilidade de assumir uma conduta baseada na melhor evidência científica disponível $^{20}$. Segundo Sackett et al. ${ }^{7}$ (2003), não há evidência empírica baseada em ensaios clínicos que mostre que o cuidado baseado em evidências produz resultados melhores para os pacientes; de outro modo, os autores observaram, em pesquisas de desfecho baseadas em populações, que pacientes cujos tratamentos são baseados em evidências apresentam melhores desfechos do que aqueles cujos tratamentos não o são ${ }^{7}$.

Portanto, para que o paciente experiencie desfechos de saúde melhores em decorrência da OBE, é necessário que o conhecimento gerado seja implementado na prática clínica diária ${ }^{9}$. A implantação de condutas baseadas em evidência, por sua vez, encontra obstáculos de naturezas variadas, incluindo a falta de conhecimento da existência das recomendações e a dificuldade de disseminação e implementação na prática. Ainda, para uma variedade de situações clínicas, não há informações devidamente selecionadas, analisadas e traduzidas em um guia de conduta clínica ou revisão sistemática. Nesse caso, cabe ao clínico o processo de sumarização da informação a partir dos estudos primários ${ }^{9}$.

A presença da OBE na prática diária é uma das exigências dos cursos de Odontologia atuais ${ }^{1}$. No entanto, algumas barreiras de ordem institucional ou ambiental e de ordem pessoal são identificadas ${ }^{20}$. Em termos institucionais, a decisão por assumir o modelo da OBE deve ser uma decisão pela confiança naquilo que se ensina, com a satisfação e a tranquilidade de oferecer o melhor tratamento disponível ${ }^{20}$, e não pode ser tomada apenas por um indivíduo, devendo envolver um esforço conjunto de todos os envolvidos no curso para a construção de uma referência de atendimento fundamentado na OBE.

Outro desafio para a implementação da OBE no meio acadêmico é o desenvolvimento da curiosidade nos acadêmicos, ou seja, o incômodo com as lacunas no seu conhecimento acerca do diagnóstico, tratamento ou prognóstico de determinado caso. Dela parte a sistematização da busca pela informação que dê conta de esclarecer sua dúvida sobre a melhor conduta ${ }^{7}$. Por óbvio que possa parecer, o estímulo à curiosidade, à elaboração de perguntas e à 
problematização não é habitual no meio acadêmico, visto que o modelo hegemônico de aula expositiva dá pouca vazão à habilidade de problematização.

Além disso, outros aspectos de ordem pessoal configuram-se em barreiras à implantação efetiva da OBE. Dentre eles, a falta de conhecimento ou o conhecimento obsoleto de muitos docentes acerca de aspectos da epidemiologia clínica que fundamenta a OBE e a tradição da disseminação da informação na odontologia pautada na opinião de experts. Também chamam a atenção as limitações no uso de banco de dados eletrônicos, o acesso aos artigos que trazem a informação sem intermediários e limitações com a língua inglesa ${ }^{20,21}$.

Diante das barreiras apontadas, algumas alternativas podem ser aventadas para cursos que queiram assumir a OBE como um fio condutor do processo de ensino-aprendizagem. Uma possibilidade pode ser a introdução de abordagens de aprendizagem baseadas em problemas, extensamente relatadas na literatura da área da saúde e pautadas na busca de soluções para problemas clínicos apresentados. De fato, qualquer abordagem guiada que torne $o$ acadêmico o protagonista do processo de busca é bem-vinda. Marshall et al. ${ }^{21}$ (2013) sugerem que oportunidades de treinamento e de suporte técnico científico a docentes são fundamentais para garantir a incorporação da OBE no currículo dos cursos de Odontologia, uma vez que muitos não tiveram contato com os fundamentos da OBE durante a graduação ou pós-graduação.

\section{Considerações finais}

A Odontologia baseada em evidências é um movimento irreversível na maneira de pensar o cuidado com a saúde. Como tal, ela deve ser exercitada pelos envolvidos no processo de ensino-aprendizagem acadêmico de forma conjunta. Esta revisão de literatura apresentou elementos para a compreensão e a construção de estratégias de busca por alternativas terapêuticas em odontologia. Sua continuação trará elementos que contribuam para a interpretação de estudos de revisão sistemática e metanálise, de modo que acadêmicos, professores e cirurgiões-dentistas possam extrair desses artigos as informações necessárias para a tomada de decisão clínica.

\section{Abstract}

Evidence-Based Dentistry (EBD) aims to rationalize the dental practice based on the best scientific evidence available, the systemized clinical experience, and the preferences and conditions of patients. Objectives: This study presents elements that establish EBD and guide the search for evidences on therapeutic interventions in Dentistry. Literature review: The translation of evidence generated by researches to the clinical applicability is key to support clinical management and is mediated by systematic literature reviews. These reviews may be performed from either in vitro primary studies or randomized controlled clinical trials (RCCTS). The search for references that present evidence about a given issue is constructed from a structured question including the type of patient/problem/population, intervention, and comparisons between alternatives and outcome. The search strategy should be applied to more than one database, necessarily including PubMed-Medline and the Cochrane library of systematic reviews and RCCTs. When the clinical question aims to solve a therapeutic doubt, the highest levels of evidence are presented by systematic reviews and RCCTs. Final considerations: $E B D$ is an irreversible movement on how to think about health care and, therefore, should be addressed in all fields of dentistry, both in graduation and post-graduation courses. This literature review presented elements for the construction of the clinical question and search strategies for therapeutic alternatives in dentistry.

Keywords: Epidemiology. Evidence-Based Dentistry. Evidence-Based Clinical Practice.

\section{Referências}

1. Brasil. CNE. Resolução CNE/CES n ${ }^{\circ}$ 3, de 19 de fevereiro de 2002. Diário Oficial da República Federativa do Brasil, Brasília, 4 de março de 2002, Seção 1, p. 10.

2. Bernardo WM. Introdução à revisão sistemática. In: Nita ME, Secoli SR, Nobre MRC, Ono-Nita SK, Campino ACC, Santi FM, et al. Avaliação de tecnologias em saúde. Porto Alegre: Artmed; 2010. p. 131-46.

3. Brasil. Ministério da Saúde. Secretaria de Ciência, Tecnologia e Insumos Estratégicos. Departamento de Ciência e Tecnologia. Diretrizes metodológicas: elaboração de revisão sistemática e metanálise de ensaios clínicos randomizados/ Ministério da Saúde, Secretaria de Ciência, Tecnologia e Insumos Estratégicos, Departamento de Ciência e Tecnologia. Brasília: Ministério da Saúde; 2012. 92 p. (Série A: Normas e Manuais Técnicos).

4. Demathé A, Silva ARS, De Carli JP, Goiato MC, Miyahara GI. Odontologia baseada em evidências: otimizando a prática e a pesquisa. RFO UPF 2012; 17(1):96-100.

5. Nadanovsky P. Odontologia baseada em evidência. In: Luiz RR, Costa AJL, Nadanovswy P. Epidemiologia e bioestatística em Odontologia. São Paulo: Atheneu; 2008. p. 375-95.

6. Torgenson DJ, Torgenson CJ. Designing randomized trials in health, education and the social sciences. New York: Palgrave MacMillan; 2008.

7. Sackett DL, Straus SE, Richardson WS, Rosenberg W, Brian Haynes R. Medicina baseada em evidências - prática e ensino. 2. ed. Porto Alegre: Artmed; 2003.

8. Morris ZS, Wooding S, Grant J. The answer is 17 years, what is the question: understanding time lags in translational research. J R Soc Med 2011; 104(12):510-20.

9. Tracy SL. From bench-top to chair-side: how scientific evidence is incorporated into clinical practice. Dent Mater 2014; 30(1):1-15.

10. Liberati A, Altman DG, Tetzlaff J, Mulrow C, Gøtzsche PC, Ioannidis JPA, et al. The PRISMA statement for reporting systematic reviews and meta-analyses of studies that evaluate healthcare interventions: explanation and elaboration. BMJ 2009; 339:b2700. DOI: 10.1136/bmj.b2700. 
11. Sutherland SE. An introduction to systematic review. J Evid Base Dent Pract 2004; 4(1):47-51.

12. Higgins JPT, Green S (editors). Cochrane Handbook for Systematic Reviews of Interventions Version 5.1.0 [updated March 2011]. The Cochrane Collaboration, 2011. Disponível em URL: www.cochrane-handbook.org.

13. Perissé ARS, Gomes MM, Nogueira SA. Revisões sistemáticas (inclusive metanálises) e diretrizes clínicas. In: Gomes MM. Medicina baseada em evidências. Rio de Janeiro: Reichmann \& Affonso; 2001. p. 131-48.

14. Martins MFM, Gomes MM. Procurando a melhor evidência clínica. In: Gomes MM. Medicina baseada em evidências. Rio de Janeiro: Reichmann \& Affonso; 2001. p. 63-80.

15. Moher D, Hopewell S, Schulz KF, Montori V, Gøtzsche PC, Devereaux PJ, et al. CONSORT 2010 Explanation and elaboration: update guidelines for reporting parallel group randomized trials. BMJ 2010; 340:c869. DOI:10.1136/bmj.c869.

16. Moher D, Pham B, Jones A, Cook DJ, Jadad AR, Moher M, et al. Does quality of reports of randomized trials affect estimates of intervention efficacy reported in meta-analyses. Lancet 1998; 352(9128):609-13.

17. Verhagen AP, de Vet HCW, de Bie RA, Boers M, van den Brandt PA. The art of quality assessment of RCTs included in systematic reviews. J Clin Epidemiol 2001; 54(7):651-4.

18. De Munck J, Mine A, Poitevin A, Van Ende A, Vivan Cardoso M, Van Landuyt KL, et al. Meta-analytical review of parameters involved in dentin bonding. J Dent Res 2012; 91(4):351-7.

19. Montagner AF, Sarkis-Onofre R, Pereira-Cenci T, Cenci MS. MMP inhibitors on dentin stability: a systematic review and meta-analysis. J Dent Res 2014; 93(8):733-43.

20. Kishore M, Panat SR, Aggarwal A, Agarwal N, Upadhyay N, Alok A. Evidence based dental care: Integrating clinical expertise with systematic research. J Clin Diagn Res 2014; 8(2):259-62.

21. Marshall TA, Straub-Morarend CL, Qian F, Finkelstein MW Perceptions and practices of dental school faculty regarding evidence-based dentistry. J Dent Educ 2013; 77(2):146-51.

\section{Endereço para correspondência:}

Sinval Adalberto Rodrigues-Junior Universidade Comunitária da Região de Chapecó Área de Ciências da Saúde - Caixa postal 1.141 Av. Senador Atílio Fontana, 591-E Efapi 89809-000 Chapecó, SC, Brasil

Telefone: (49) 9942-3884

E-mail: rodriguesjunior.sa@unochapeco.edu.br

Recebido: 07/07/2016. Aceito: 09/09/2016. 